\title{
Correction to: Respiratory infections are temporally associated with initiation of type 1 diabetes autoimmunity: the TEDDY study
}

\author{
Maria Lönnrot ${ }^{1,2} \cdot$ Kristian F. Lynch ${ }^{3}$ - Helena Elding Larsson ${ }^{4,5}$. \\ Åke Lernmark ${ }^{5,6}$ • Marian J. Rewers $^{7}$ • Carina Törn ${ }^{4,5}$ • Brant R. Burkhardt ${ }^{3}$. \\ Thomas Briese $^{8,9}$ • William A. Hagopian ${ }^{10}$ • Jin-Xiong She ${ }^{11}$ • Olli G. Simell ${ }^{12,13}$. \\ Jorma Toppari $^{14,15}$ • Anette-G. Ziegler ${ }^{16,17}$ - Beena Akolkar ${ }^{18}$. \\ Jeffrey P. Krischer ${ }^{3} \cdot$ Heikki Hyöty ${ }^{2,19}$ • on behalf of the TEDDY Study Group
}

Published online: 27 October 2017

(C) Springer-Verlag GmbH Germany 2017

\section{Correction to: Diabetologia \\ https://doi.org/10.1007/s00125-017-4365-5}

The authors regret that the SNP in $S H 2 B 3$ was incorrectly referred to as rs3184505 instead of rs3184504 on both mentions in this paper (Methods section and Table 1).

The online version of the original article can be found at https://doi.org/ 10.1007/s00125-017-4365-5

Maria Lönnrot

maria.lonnrot@uta.fi

1 Department of Dermatology, Tampere University Hospital, Teiskontie 35, 33521 Tampere, Finland

2 Faculty of Medicine and Life Sciences, University of Tampere, Tampere, Finland

3 Health Informatics Institute, Morsani College of Medicine, University of South Florida, Tampa, FL, USA

4 Department of Clinical Sciences Malmö, Clinical Research Centre (CRC), Lund University, Malmö, Sweden

5 Skåne University Hospital (SUS), Malmo, Sweden

6 Department of Clinical Sciences Malmö, Lund University Clinical Research Centre (CRC), Malmö, Sweden

7 Barbara Davis Center for Childhood Diabetes, University of Colorado, Aurora, CO, USA

8 Center for Infection and Immunity, Mailman School of Public Health, Columbia University, New York, NY, USA
9 Department of Epidemiology, Mailman School of Public Health, Columbia University, New York, NY, USA

10 Pacific Northwest Diabetes Research Institute, Seattle, WA, USA

11 Center for Biotechnology and Genomic Medicine, Medical College of Georgia, Augusta University, Augusta, GA, USA

12 Research Centre of Applied and Preventive Cardiovascular Medicine, Faculty of Medicine, University of Turku, Turku, Finland

13 Department of Paediatrics and Adolescent Medicine, Faculty of Medicine, University of Turku, Turku, Finland

14 Department of Physiology, Institute of Biomedicine, University of Turku, Turku, Finland

15 Department of Paediatrics, Turku University Hospital, Turku, Finland

16 Forschergruppe Diabetes e.V, Neuherberg, Germany

17 Institute of Diabetes Research, Helmholtz Zentrum München, Munich, Germany

18 National Institute of Diabetes \& Digestive \& Kidney Diseases, Bethesda, MD, USA

19 Fimlab Laboratories, Pirkanmaa Hospital District, Tampere, Finland 